\title{
A Note on Strongly Lower Semi-Continuous Functions and the Induced Fuzzy Topological Space Generated by Them
}

\author{
Apu Kumar Saha \& Debasish Bhattacharya \\ Department of Mathematics, National Institute of Technology, \\ Agartala, Jirania-799055, Tripura, India. \\ E-mail: apusaha_nita@yahoo.co.in
}

\begin{abstract}
A new class of functions called strongly lower semi-continuous (SLSC) functions is defined and its properties are studied. It is shown that the arbitrary supremum and finite infimum of SLSC functions are again SLSC. Using these functions, an induced fuzzy topological space, called s-induced fuzzy topological space on a topological space $(\mathrm{X}, \mathrm{T})$, is introduced. Moreover, some incorrect results on fuzzy topological spaces obtained previously by some authors are identified and modified accordingly. Examples of the newly defined induced space are given and their various properties are investigated. Interrelationships between a fuzzy topological space $(X, \mathcal{F})$ and the s-induced fuzzy topological space generated by the crisp members of $\mathcal{F}$ are examined. In this process, different lower semi-continuities and induced fuzzy spaces generated by them have been defined in a general set up and their few properties have been studied.
\end{abstract}

Keywords: fuzzy topological space; regular $F_{\sigma^{-}}$subset; s-induced fuzzy topological space; $S$-LSC function; SLSC function; topology.

\section{$1 \quad$ Introduction}

In 1968, C.L. Chang [1] put forward the concept of fuzzy topological space as a very natural generalization of topological space in the literature, after the discovery of fuzzy sets by L.A. Zadeh [2] in 1965, as follows.

Definition 1.1: Let $X$ be a non-empty set. A family $\mathcal{F}$ of fuzzy subsets of $X$ is called fuzzy topology on $\mathrm{X}$ if

1. $0,1 \in \mathscr{F}$;

2. arbitrary suprema of members of $\mathcal{F}$ are in $\mathscr{F}$;

3. finite infima of members of $\mathcal{F}$ are in $\mathcal{F}$.

The pair $(\mathrm{X}, \mathcal{F})$ is called fuzzy topological space. The members of $\mathcal{F}$ are called fuzzy open sets. The complements of fuzzy open sets are called fuzzy closed sets.

Received June $26^{\text {th }}, 2012,1^{\text {st }}$ Revision November $26^{\text {th }}, 2012,2^{\text {nd }}$ Revision February $13^{\text {th }}, 2013$, Accepted for publication February $14^{\text {th }}, 2013$.

Copyright $\odot 2013$ Published by ITB Journal Publisher, ISSN: 2337-5760, DOI: 10.5614/j.math.fund.sci.2013.45.1.6 
R. Lowen [3], in 1976 suggested an alternative and more natural definition of fuzzy topology (called stratified fuzzy topology) for achieving more results which are compatible with the general case in topology. Lowen pointed out that the above definition of fuzzy topology suffers from a serious drawback, i.e., constant functions between fuzzy topological spaces are not necessarily continuous, which is in contrary to our perception that all constant functions are necessarily continuous in topological spaces. To overcome this difficulty Lowen introduced the concept of stratified fuzzy topology making all constant functions (and not only 0 and 1) open. Thus the stratified fuzzy topology due to Lowen is more general than that of Chang.

Definition 1.2: Let $X$ be a non-empty set. A family $\mathcal{F}$ of fuzzy subsets of $X$ is called stratified fuzzy topology on $\mathrm{X}$ if

1. $\alpha \in \mathcal{F}$, where $\alpha$ is a constant function taking the constant value $\alpha \in \mathrm{I}$;

2. arbitrary suprema of members of $\mathcal{F}$ are in $\mathscr{F}$;

3. finite infima of members of $\mathcal{F}$ are in $\mathcal{F}$.

The pair $(\mathrm{X}, \mathcal{F})$ is called stratified fuzzy topological space. The members of $\mathcal{F}$ are called fuzzy open sets. The complements of fuzzy open sets are called fuzzy closed sets.

There is a natural way to associate a fuzzy topology $\mathcal{F}$ on a set $\mathrm{X}$ to a given topology $\mathrm{T}$ on $\mathrm{X}$ by means of the collection of all lower semi-continuous (LSC) functions from $(X, T)$ to the unit closed interval $I=[0,1]$. Weiss [4] called this fuzzy topology on $\mathrm{X}$ an induced fuzzy topology on $(\mathrm{X}, \mathrm{T})$ providing a concrete class of examples of the fuzzy topologies. Various properties of fuzzy sets in the presence of an induced fuzzy topology were also studied by Weiss, who utilized it to prove a generalization to fuzzy sets of the Schauder-Tychonoff theorem, which asserts that "every continuous self-mapping of a compact convex subset of a locally convex linear topological space has a fixed point". He further proved a revised version of the fuzzy separation theorem of Zadeh for appropriate fuzzy subsets of a linear topological space, employing the notion of induced fuzzy topology. Thus, induced fuzzy topological spaces may be used as a tool to prove the various complicated theorems of fuzzy topological spaces in a much easier way.

In the recent past, the concept of induced fuzzy topological space have been further studied by some authors using stronger forms of LSC functions viz. completely LSC and $\delta$-LSC functions, as well as weaker forms such as $\alpha$-LSC etc. In [5], Bhaumik and Mukherjee introduced completely induced fuzzy topological space with the help of completely LSC (CLSC) functions and Mukherjee and Halder [6] introduced $\delta$-induced fuzzy topological space with 
the help of $\delta$-LSC functions, and various properties of the respective spaces were studied. It was observed that the various properties of the completely induced fuzzy topological space studied in $[7,8]$ are based on Theorem 3.2 of [5], which states that "a fuzzy subset A in completely induced fuzzy topological space $(X, C(T))$ is open iff for each $r \in I$, the strong $r$-cut $\sigma_{r}(A)$ is regular open in the topological space $(\mathrm{X}, \mathrm{T})$ ". But, incidentally, it has been noticed that the above result is not always valid. In fact, the modified version of the theorem would be "a fuzzy subset A in completely induced fuzzy topological space (X, $C(T)$ ) is open iff for each $r \in I$, the strong $r$-cut $\sigma_{r}(A)$ is a union of regular open sets in the topological space (X, T)", which is shown in Theorem 3.7 of this paper. Actually, Theorem 3.2 of [5] is a consequence of Theorem 1 of [5] which states that "the necessary and sufficient condition for a real valued function $\mathrm{f}$ to be CLSC is that for all $a \in R$, the set $\{x \in X: f(x)>a\}$ is regular open (or equivalently $\{\mathrm{x} \in \mathrm{X}: \mathrm{f}(\mathrm{x}) \leq \mathrm{a}\}$ is regular closed)". That this is not true can be seen in Note 3.11 of this paper. The modified version of this theorem would be "the necessary and sufficient condition for a real valued function $\mathrm{f}$ to be CLSC is that for all $a \in R$, the set $\{x \in X: f(x)>a\}$ is a union of regular open sets". Similar modifications are also necessary for $\delta$-LSC functions and $\delta$-induced fuzzy topological space in [6]. These observations challenge the entire study of the CLSC function and $\delta$-LSC functions together with the induced fuzzy spaces generated by them.

Again, in 1987, Monsef and Ramadan [9] introduced the concept of fuzzy supra topology as a generalization of fuzzy topology, and in 1998, Mukherjee [10] defined an induced fuzzy supra topological space viz. $\alpha$-induced fuzzy supra topological space with the help of $\alpha$-LSC functions. But, modification as suggested above is again necessary in respect of Result-4.2 (i) of [10].

Thus, it is clear that the properties of different induced fuzzy topological spaces, and the induced fuzzy supra-topological spaces mentioned above which were introduced after Weiss [4], were not studied correctly and thus need further, indepth study.

The motivation of the present treatise is to search for a stronger version of the LSC function that is capable of generating an induced fuzzy topological space on a topological space $(\mathrm{X}, \mathrm{T})$ and to study the properties of that function as well as that of the induced fuzzy topological space in the correct form, and to check whether the properties of induced fuzzy topological space studied by Weiss [4] are also valid in the present setup or not. Secondly, our aim is to study the interrelationships between a fuzzy topological space $(\mathrm{X}, \mathfrak{F})$ and the newly defined induced space on $(\mathrm{X}, \mathrm{T})$, where $\mathrm{T}=\mathfrak{F} \cap 2^{\mathrm{X}}$ is the crisp part of $\mathcal{F}$. 
Again, we have seen that all the stronger (or weaker) forms of LSC functions (found in the literature) may fail to generate induced fuzzy topological space, for example, the regular LSC (RLSC) functions [11], normal LSC (NLSC) functions [12], etc. It was observed that only the countable supremum of RLSC functions is RLSC but the arbitrary supremum of RLSC functions may not be so [7]. With the help of RLSC functions, the authors have introduced a new fuzzy topological structure and called it r-countably induced fuzzy topological space or rc-induced fts [13]. The authors also investigated the cardinal extension of the aforementioned structure [11]. On the other hand, even the finite suprema of NLSC functions may not be NLSC. Therefore, the NLSC functions fail to form an induced fuzzy topology on a given topological space, although finite infima of NLSC functions are NLSC. With the help of NLSC functions a generalized fuzzy topology, namely n-infy induced fuzzy topological space, has been defined [14] by the authors and its various properties have been investigated. It may be seen that the generalized forms of fuzzy topological structures have very important applications in many areas of science and technology, such as quantum particles physics, particularly in connection with string theory and $\varepsilon^{\infty}$ theory [15-17].

To start with, we recall that a function $\mathrm{f}: \mathrm{X} \rightarrow \mathrm{R}$ is LSC at a point $\mathrm{x}_{0} \in \mathrm{X}$, if for each $\varepsilon>0$ there exists a neighborhood (nbd.) of $\mathrm{x}_{0}$, say $\mathrm{N}\left(\mathrm{x}_{0}\right)$, such that for all $x \in N\left(x_{0}\right), f(x)>f\left(x_{0}\right)-\varepsilon[18]$. Replacing the $n b d$. by stronger forms of open sets we may find various stronger forms of LSC functions. For example, we may replace the nbd. by regular open nbd. to obtain completely LSC functions [5], $\delta$ open nbd. to obtain the $\delta$-LSC functions [6], $\alpha$-open nbd. to obtain $\alpha$-LSC functions [10], etc. Apart from these, one may take regular $F_{\sigma}$-nbd., co-zero nbd. etc. of the point instead of nbd. $\mathrm{N}\left(\mathrm{x}_{0}\right)$. In the present paper, we use regular $F_{\sigma}$-nbd. of the point considered to the define strongly LSC function that is capable of generating induced fuzzy topological space. By using co-zero subsets, another induced fuzzy topological structure may be defined and studied in the future.

\section{Strongly Lower Semi-Continuous Function}

Before defining the strongly lower semi-continuous (SLSC) function let us recall the following concepts.

J. E. Mack [19] introduced the concept of regular $G_{\delta}$-subset and regular $F_{\sigma^{-}}$ subset as follows.

Definition 2.1. A subset $\mathrm{H}$ of a topological space $\mathrm{X}$ is called a regular $\mathrm{G}_{\delta}$-subset if $\mathrm{H}$ is an intersection of a sequence of closed sets whose interiors contain $\mathrm{H}$. 
Equivalently, if $H=\bigcap_{i} G_{i}=\bigcap_{i} c_{X} G_{i}$ for $i \in N$, where each $G_{i}$ is open in $X$, then $\mathrm{H}$ is a regular $\mathrm{G}_{\delta}$-subset of $\mathrm{X}$.

Definition 2.2. A subset $K$ of a topological space $X$ is called a regular $F_{\sigma}$-subset if $\mathrm{K}$ is a union of a sequence of open sets whose closures are contained in $\mathrm{K}$. Equivalently, if $K=\bigcup_{i} F_{i}=\bigcup_{i}$ int $_{X} F_{i}$ for $i \in N$, where each $F_{i}$ is closed in $X$, then $\mathrm{K}$ is a regular $\mathrm{F}_{\sigma}$-subset of $\mathrm{X}$.

It can be easily verified that the complement of a regular $\mathrm{G}_{\delta}$-subset is a regular $\mathrm{F}_{\sigma}$-subset and vice versa. Also every regular $\mathrm{G}_{\delta}$-subset (resp. regular $\mathrm{F}_{\sigma}$-subset) being the countable intersection (resp. countable union) of closed (resp. open) subsets of $\mathrm{X}$ is closed (resp. open) in $\mathrm{X}$. The collection of all regular $\mathrm{G}_{\delta}$-subsets (resp. regular $\mathrm{F}_{\sigma}$-subsets) of a space $\mathrm{X}$ is closed under finite union and countable intersections (resp. countable union and finite intersection) [7].

As mentioned earlier using lower semi-continuous functions and its stronger forms many researchers have attempted to define topologically generated fuzzy topological spaces. All these functions can be seen to have been generated from a very general setup. With this aim we introduce the concept of $S$-lower semicontinuity. The existing LSC functions and other stronger forms come as a particular case of the defined generalized function.

Definition 2.3. Let $S$ be a family of subsets of a given set $\mathrm{X}$ equipped with a topology $\mathrm{T}$, which is closed under finite intersections and contains $\mathrm{X}$ and $\phi$. Then, a function $\mathrm{f}: \mathrm{X} \rightarrow \mathrm{R}$ is said to be an $S$-lower semi-continuous function if for every $\mathrm{x} \in \mathrm{X}$ and every $\varepsilon>0$, there exists some $\mathrm{S} \in S$, which contains $\mathrm{x}$, and for every $y \in S$, it follows that $f(y)>f(x)-\varepsilon$.

The set $S \in S$ containing a point $\mathrm{x} \in \mathrm{X}$ will be called $S$-nbd. of x. Also the members of $S$ are called $S$-sets and their complements will be referred to as $S^{\text {c }}$ sets.

If $S$ be the collection of open, regular open, regular $\mathrm{F}_{\sigma}, \delta$-open, etc. subsets of $\mathrm{X}$, then the corresponding $S$-lower semi-continuous ( $S$-LSC) functions are termed as lower semi-continuous (LSC), CLSC, $\delta$-LSC, SLSC etc. functions, respectively.

In this paper, we will study induced fuzzy topological spaces generated by SLSC functions where the $S$-sets are regular $\mathrm{F}_{\sigma}$-subsets of X. 
Definition 2.4. A function $\mathrm{f}:(X, \mathrm{~T}) \rightarrow(\mathrm{R}, \mathrm{u})$ is said to be strongly lower semicontinuous or SLSC function at a point $\mathrm{x}_{0} \in \mathrm{X}$, if for each $\varepsilon>0$, there exists a regular $\mathrm{F}_{\sigma}$-nbd. $\mathrm{N}\left(\mathrm{x}_{0}\right)$, such that $\mathrm{x} \in \mathrm{N}\left(\mathrm{x}_{0}\right)$ implies $\mathrm{f}(\mathrm{x})>\mathrm{f}\left(\mathrm{x}_{0}\right)-\varepsilon$.

Next we define the strongly continuous function as follows.

Definition 2.5. A function $\mathrm{f}:(\mathrm{X}, \mathrm{T}) \rightarrow(\mathrm{R}, \mathrm{u})$ is said to be strongly continuous at a point $\mathrm{x}_{0} \in \mathrm{X}$, if for each $\varepsilon>0$, there exists a regular $\mathrm{F}_{\sigma}$-nbd. $\mathrm{N}\left(\mathrm{x}_{0}\right)$, such that $\mathrm{x} \in$ $\mathrm{N}\left(\mathrm{x}_{0}\right)$ implies $\mathrm{f}\left(\mathrm{x}_{0}\right)-\varepsilon<\mathrm{f}(\mathrm{x})<\mathrm{f}\left(\mathrm{x}_{0}\right)+\varepsilon$.

A function $\mathrm{f}$ is said to be strongly continuous (respectively SLSC) on the space $\mathrm{X}$ if the function is strongly continuous (respectively SLSC) at all the points of $\mathrm{X}$.

To start with, we first investigate some properties of the generalized $S$-LSC functions which will be also valid for other forms of LSC functions mentioned above.

Theorem 2.6. The necessary and sufficient condition for a real valued function f on $\mathrm{X}$ to be $S$-LSC is that for all a $\in \mathrm{R}$, the set $\{\mathrm{x} \in \mathrm{X}: \mathrm{f}(\mathrm{x})>$ a $\}$ is a union of $S$ sets.

Proof. Let $\mathrm{f}$ be an $S$-LSC function and $\mathrm{x}_{0} \in \mathrm{X}$. Then for each $\varepsilon>0$, there exists an $S$-nbd. $\mathrm{S}\left(\mathrm{x}_{0}\right)$ such that for $\mathrm{x} \in \mathrm{S}\left(\mathrm{x}_{0}\right), \mathrm{f}(\mathrm{x})>\mathrm{f}\left(\mathrm{x}_{0}\right)-\varepsilon$. Again let $\mathrm{A}=\{\mathrm{x}: \mathrm{f}(\mathrm{x})>$ a) and let $\mathrm{y} \in \mathrm{A}$, i.e., $\mathrm{f}(\mathrm{y})>\mathrm{a}$. Now, we choose $\varepsilon>0$ such that $\mathrm{f}(\mathrm{y})-\varepsilon>$ a. Since f is $S$-LSC, there exists an $S$-nbd. $\mathrm{S}(\mathrm{y})$ of y such that for all $\mathrm{x} \in \mathrm{S}(\mathrm{y}), \mathrm{f}(\mathrm{x})>$

$\mathrm{f}(\mathrm{y})-\varepsilon>\mathrm{a}$. Thus, for all $\mathrm{x} \in \mathrm{S}(\mathrm{y}), \mathrm{f}(\mathrm{x})>\mathrm{a}$ and therefore $\mathrm{S}(\mathrm{y}) \subseteq \mathrm{A}$. Now, $\mathrm{y} \in \mathrm{S}(\mathrm{y})$ $\subseteq \mathrm{A}$, therefore $\bigcup\{\mathrm{y}\} \subseteq \bigcup S(\mathrm{y}) \subseteq \mathrm{A}$, i.e., $\mathrm{A} \subseteq \bigcup S(\mathrm{y}) \subseteq \mathrm{A}$ and hence $\mathrm{A}=\bigcup \mathrm{S}(\mathrm{y})$. Thus, $\mathrm{A}=\{\mathrm{x}: \mathrm{f}(\mathrm{x})>\mathrm{a}\}$ is a union of members of $S$.

Conversely, let $\{\mathrm{x}: \mathrm{f}(\mathrm{x})>\mathrm{a}\}$ be a union of $S$-sets for each a $\in \mathrm{R}$ and $\mathrm{x}_{0} \in \mathrm{X}$. For $\varepsilon$ $>0, \mathrm{f}\left(\mathrm{x}_{0}\right)-\varepsilon=\mathrm{b}$ (say). So, the set B $=\left\{\mathrm{x}: \mathrm{f}(\mathrm{x})>\mathrm{f}\left(\mathrm{x}_{0}\right)-\varepsilon\right\}$ is a union of $S$-sets. But $\mathrm{x}_{0} \in \mathrm{B}$, so, there exists at least one $S$-set, say $\mathrm{S}_{0}$, of the above union which contains $\mathrm{x}_{0}$. Thus, for $\mathrm{x} \in \mathrm{S}_{0}, \mathrm{f}(\mathrm{x})>\mathrm{f}\left(\mathrm{x}_{0}\right)-\varepsilon$. Hence, $\mathrm{f}$ is $S$-LSC.

Similarly, it can be proved that for a strongly continuous function $\mathrm{f}: \mathrm{X} \rightarrow \mathrm{R}$, both the sets $\{\mathrm{x} \in \mathrm{X}: \mathrm{f}(\mathrm{x})>\mathrm{a}\}$ and $\{\mathrm{x} \in \mathrm{X}: \mathrm{f}(\mathrm{x})<\mathrm{a}\}$ are a union of $S$-sets.

The following theorem, which is actually a modification of Theorem 1 of [5], follows as a corollary of Theorem 2.6. That Theorem 1 of [5] is not valid can be seen in Example 3.11. 
Theorem 2.7. The necessary and sufficient condition for a real valued function $f$ to be CLSC is that for all $a \in R$, the set $\{x \in X: f(x)>a\}$ is a union of regular open subsets of $\mathrm{X}$.

Corollary 2.8. A function $\mathrm{f}$ from a topological space $(X, T)$ into the space (R, $\left.\sigma_{1}\right)$, where $\sigma_{1}=\{(\mathrm{r}, \infty): \mathrm{r} \in \mathrm{R}\}$, is $S$-LSC function iff the inverse image of any open subset of $\left(\mathrm{R}, \sigma_{1}\right)$ is a union of $S$-sets.

Remark 2.9. If $\mathrm{f}$ is $S$-LSC on $\mathrm{X}$, then the set $\{\mathrm{x} \in \mathrm{X}: \mathrm{f}(\mathrm{x}) \leq \mathrm{a}\}$ is an intersection of $S^{\mathrm{c}}$-sets.

Various properties of $S$-LSC functions are studied in Theorems 2.10-2.14.

Theorem 2.10. The characteristic function of an $S$-set of $S$ is $S$-LSC.

Proof. Let A $\subseteq \mathrm{X}$ be an $S$-set. Let us now define the characteristic function $\mu_{\mathrm{A}}$ : $X \rightarrow\{0,1\}$ such that

$$
\begin{aligned}
\mu_{\mathrm{A}}(\mathrm{x}) & =1, \text { if } \mathrm{x} \in \mathrm{A} \\
& =0, \text { if } \mathrm{x} \in \mathrm{X}-\mathrm{A} .
\end{aligned}
$$

We are to show that $\mu_{\mathrm{A}}$ is $S$-LSC, i.e., the set $\mathrm{B}=\left\{\mathrm{x}: \mu_{\mathrm{A}}(\mathrm{x})>\right.$ a $\}$ is a union of $S$ sets for each $\mathrm{a} \in \mathrm{R}$.

Case-I. If a $<0$, then clearly, B $=\mathrm{X}$, which is an $S$-set and hence can be expressed as a union of $S$-sets.

Case-II. If $0 \leq \mathrm{a}<1$, then clearly, B $=\mathrm{A}$, which is an $S$-set and hence is a union of $S$-sets.

Case-III. If a $\geq 1$, then clearly, B $=\phi$, which is an $S$-set and hence is a union of $S$-sets.

In order to show that the newly defined $S$-LSC function actually induces a fuzzy topology on $\mathrm{X}$, we need to verify that the arbitrary supremum and finite infimum of $S$-LSC functions are again $S$-LSC. Also, the constant functions are $S$-LSC as well.

Theorem 2.11. The arbitrary supremum of $S$-LSC functions is $S$-LSC. Proof. Let $\left\{\mathrm{f}_{\alpha}\right\}_{\alpha \in \Lambda}$ be an arbitrary family of $S$-LSC functions from X to R. We are to show that $\mathrm{g}=\sup _{\alpha}\left(\mathrm{f}_{\alpha}\right)$ is also $S$-LSC. 
Let $\mathrm{x}_{0} \in \mathrm{X}$ and $\varepsilon>0$ be given, so $\mathrm{g}\left(\mathrm{x}_{0}\right)=\sup \mathrm{f}_{\alpha}\left(\mathrm{x}_{0}\right)$. Then there exists some $\beta$ such that $\mathrm{f}_{\beta}\left(\mathrm{x}_{0}\right)>\mathrm{g}\left(\mathrm{x}_{0}\right)-\varepsilon$. Therefore, we can choose $\varepsilon^{\prime}>0$, such that $\mathrm{f}_{\beta}\left(\mathrm{x}_{0}\right)-\varepsilon^{\prime}$ $>\mathrm{g}\left(\mathrm{x}_{0}\right)-\varepsilon$. Since, $\mathrm{f}_{\beta}$ is $S$-LSC at $\mathrm{x}_{0}$, there exists an $S$-nbd. $\mathrm{S}_{\beta}\left(\mathrm{x}_{0}\right)$ such that $\mathrm{f}_{\beta}(\mathrm{x})>\mathrm{f}_{\beta}\left(\mathrm{x}_{0}\right)-\varepsilon^{\prime}$ for all $\mathrm{x} \in \mathrm{S}_{\beta}\left(\mathrm{x}_{0}\right)$. Therefore, for all $\mathrm{x} \in \mathrm{S}_{\beta}\left(\mathrm{x}_{0}\right)$ we have $\mathrm{f}_{\beta}(\mathrm{x})>$ $\mathrm{f}_{\beta}\left(\mathrm{x}_{0}\right)-\varepsilon^{\prime}>\mathrm{g}\left(\mathrm{x}_{0}\right)-\varepsilon$.

Finally, sup $\mathrm{f}_{\alpha}(\mathrm{x}) \geq \mathrm{f}_{\beta}(\mathrm{x})$. Hence for $\varepsilon>0$, there exists an $S$-nbd. $\mathrm{S}_{\beta}\left(\mathrm{x}_{0}\right)$ such that for all $\mathrm{x} \in \mathrm{S}_{\beta}\left(\mathrm{x}_{0}\right)$, sup $\mathrm{f}_{\alpha}(\mathrm{x})>\sup \mathrm{f}_{\alpha}\left(\mathrm{x}_{0}\right)-\varepsilon$, i.e. $\mathrm{g}(\mathrm{x})>\mathrm{g}\left(\mathrm{x}_{0}\right)-\varepsilon$. Hence $\mathrm{g}$ is $S$ LSC.

Theorem 2.12. Finite infimum of $S$-LSC functions is $S$-LSC.

Proof. Let $\mathrm{f}_{1}, \mathrm{f}_{2} \ldots \mathrm{f}_{\mathrm{n}}$ be a finite collection of $S$-LSC functions from X to R. We are to show that $\mathrm{h}=\inf \left(\mathrm{f}_{\mathrm{i}}\right), \mathrm{i}=1,2 \ldots \mathrm{n}$ is $S$-LSC.

Let $\mathrm{x}_{0} \in \mathrm{X}$. Since each $\mathrm{f}_{\mathrm{i}}$ is $S$-LSC, for every $\varepsilon>0$, there exists a $S$-nbd. $\mathrm{S}_{\mathrm{i}}\left(\mathrm{x}_{0}\right)$ such that $\mathrm{f}_{\mathrm{i}}(\mathrm{x})>\mathrm{f}_{\mathrm{i}}\left(\mathrm{x}_{0}\right)-\varepsilon$. Again, the intersection of a finite number of $S$-sets is an $S$-set, so we have $\bigcap_{\mathrm{i}} \mathrm{S}_{\mathrm{i}}\left(\mathrm{x}_{0}\right), \mathrm{i}=1,2, \ldots, \mathrm{n}$, is an $S$-nbd. of $\mathrm{x}_{0}$. Now, if $\mathrm{x} \in$ $\bigcap_{\mathrm{i}} \mathrm{S}_{\mathrm{i}}\left(\mathrm{x}_{0}\right)$, then for all $\mathrm{i}, \mathrm{f}_{\mathrm{i}}(\mathrm{x})>\mathrm{f}_{\mathrm{i}}\left(\mathrm{x}_{0}\right)-\varepsilon$. i.e., $\inf \mathrm{f}_{\mathrm{i}}(\mathrm{x})>\inf \left(\mathrm{f}_{\mathrm{i}}\left(\mathrm{x}_{0}\right)-\varepsilon\right)$, i.e., $\mathrm{h}(\mathrm{x})>$ $\mathrm{h}\left(\mathrm{x}_{0}\right)-\varepsilon$. Hence, h is $S$-LSC.

The corresponding results on CLSC, $\delta$ LSC and $\alpha$-LSC functions respectively given in $[6,10,19]$ are the corollaries of Theorems 2.11 and 2.12 .

Following Theorems 2.13, 2.14 and 2.15, we show that $S$-LSC functions are also closed under usual addition, multiplication and scalar multiplication operations.

Theorem 2.13. If f and $\mathrm{g}$ are two $S$-LSC functions on a space $\mathrm{X}$, then so is $\mathrm{f}+\mathrm{g}$.

Theorem 2.14. If $\mathrm{f}$ and $\mathrm{g}$ are two $S$-LSC functions on a space $\mathrm{X}$, then so is f.g.

Theorem 2.15. If $\mathrm{f}$ is $S$-LSC, then for any positive real number a, af is also $S$-LSC.

The above theorems can be proved by using standard techniques.

Next, we study the properties of SLSC functions which are special types of $S$-LSC functions when the $S$-sets are regular $\mathrm{F}_{\sigma}$-subsets of X. That every continuous function is SLSC is shown in the example below. 
Example 2.16. We know that if a function $\mathrm{f}: \mathrm{X} \rightarrow \mathrm{R}$ is continuous, then the set $\{x: f(x)>a\}, a \in R$, is a co-zero set (3.5, [20]). Again, every co-zero set is a regular $F_{\sigma^{-}}$-subset [6]. So, the sets of the form $\{x: f(x)>a\} a \in R$ are regular $F_{\sigma^{-}}$ subsets and hence unions of regular $\mathrm{F}_{\sigma}$-subsets. Thus $\mathrm{f}$ is SLSC.

Again, since every continuous function is SLSC and every constant function is continuous, therefore every constant function is SLSC.

Note 2.17. Clearly, continuity implies SLSC implies LSC. We cite two examples to show that the reverse implications are not true.

Example 2.18. Let $\mathrm{X}=[-1,1]$ and $\mathrm{A}=(0,1]$. Then $\mathrm{A}$ is a regular $\mathrm{F}_{\sigma}$-subset, since $A=\bigcup_{n} F_{n}=\bigcup_{n} \operatorname{int}_{X} F_{n}$, where $F_{n}=\left[\frac{1}{n}, 1\right]$. Now let us define a function $\mathrm{f}: \mathrm{X} \rightarrow \mathrm{I}$ such that

$$
\begin{aligned}
\mathrm{f}(\mathrm{x}) & =1, \text { if } \mathrm{x} \in \mathrm{A} \\
& =0, \text { if } \mathrm{x} \in \mathrm{X}-\mathrm{A} .
\end{aligned}
$$

So, $\mathrm{f}$ is an SLSC function (by Theorem 2.10). Let us now consider an open set B of I, given by $B=\left(-\frac{1}{3}, \frac{1}{3}\right)$. Therefore, $f^{-1}(B)=X-A$, which is not open in $X$. Hence, $\mathrm{f}$ is not continuous.

Thus, every continuous function is SLSC, but the converse is not true.

Example 2.19. Let $X=\{a, b, c\}$ be a set and $T=\{\phi, X,\{a\},\{b\},\{a, b\}\}$ be a topology defined on $X$. Then, the set $\{a, b\}$ is open but not a regular $F_{\sigma}$-subset, since $\{a, b\}$ cannot be expressed as a union of closed sets. Let us now define $f$ : $\mathrm{X} \rightarrow$ I by

$$
\begin{aligned}
f(x) & =1, \text { if } x \in\{a, b\} \\
& =0 \text {, otherwise. }
\end{aligned}
$$

Then $\mathrm{f}$ is LSC, because sets of the form $\{\mathrm{x}: \mathrm{f}(\mathrm{x})>\mathrm{r}\} \mathrm{r} \in \mathrm{R}$, are all open in X. But f fails to be SLSC because the set $\{\mathrm{x}: \mathrm{f}(\mathrm{x})>1 / 2\}=\{\mathrm{a}, \mathrm{b}\}$ is not a union of $S$ sets.

Thus, LSC does not imply SLSC.

Now, we present another characterization of SLSC functions. 
Theorem 2.20. A function $f: X \rightarrow R$ is an SLSC function iff $f^{*}=f$, where the function $\mathrm{f}^{*}(\mathrm{x})=\sup _{\mathrm{N}(\mathrm{x})} \inf _{\mathrm{y} \in \mathrm{N}(\mathrm{x})} \mathrm{f}(\mathrm{y}), \mathrm{N}(\mathrm{x})$ being a regular $\mathrm{F}_{\sigma^{-}}$-subset containing $\mathrm{x}$.

Proof. Let $\mathrm{f}: \mathrm{X} \rightarrow \mathrm{R}$ be an SLSC function. From the construction of $\mathrm{f}^{*}$, it is clear that, for all $x \in X, f^{*}(x) \leq f(x)$, i.e.

$$
\mathrm{f}^{*} \leq \mathrm{f}
$$

To prove the reverse relation, we note that for $\mathrm{x} \in \mathrm{X}$ and $\varepsilon>0$, there exists a regular $F_{\sigma}$-nbd., say $N(x)$, of $x$ such that $N(x) \subseteq\{y: f(y)>f(x)-\varepsilon\}$. Thus, $\inf _{y \in N(x)} f(y) \geq f(x)-\varepsilon$, which in turn, implies that $\sup _{N(x)} \inf _{y \in N(x)} f(y) \geq f(x)-\varepsilon$.

Since $\varepsilon>0$ is arbitrary, we have $\sup _{\mathrm{N}(\mathrm{x})} \inf _{\mathrm{y} \in \mathrm{N}(\mathrm{x})} \mathrm{f}(\mathrm{y}) \geq \mathrm{f}(\mathrm{x})$, i.e. $\mathrm{f}^{*}(\mathrm{x}) \geq \mathrm{f}(\mathrm{x})$ and hence

$$
f^{*} \geq f
$$

From (1) and (2), we have $f^{*}=f$.

Conversely, let $f^{*}(x)=f(x)$. Then, by definition, for $\varepsilon>0$, inf $f_{y \in N(x)} f(y)>f(x)-$ $\varepsilon$, for some regular $\mathrm{F}_{\sigma}$-subset $\mathrm{N}(\mathrm{x})$ containing $\mathrm{x}$. Thus, $\mathrm{f}(\mathrm{y})>\mathrm{f}(\mathrm{x})-\varepsilon$ for all $\mathrm{y} \in \mathrm{N}(\mathrm{x})$. Therefore, for each $\mathrm{x} \in \mathrm{X}$ and for $\varepsilon>0$, there exists a regular $\mathrm{F}_{\sigma^{-}}$ neighborhood $N(x)$ of $x$ such that for all $y \in N(x), f(y)>f(x)-\varepsilon$. Hence, $f$ is SLSC.

\section{$3 \quad$ Strongly Induced Fuzzy Topological Spaces}

In this section, we introduce a generalized induced fuzzy topological space with the help of $S$-LSC functions. Also, as a particular case of this fuzzy topology, strongly induced fuzzy topological space, is introduced using the class of SLSC functions. Additionally, properties of this space are also studied. We start with the following theorem.

Theorem 3.1. Let $(\mathrm{X}, \mathrm{T})$ be a topological space. The family of all $S$-LSC functions from the space $(X, T)$ to the closed unit interval $I=[0,1]$ forms a fuzzy topology on X.

Proof. Let $\mathscr{H}$ be the collection of all $S$-LSC functions from a topological space $(X, T)$ to the closed unit interval I. Now, let $\mathrm{t} \in[0,1]$, then $\mathrm{t} 1_{\mathrm{X}} \in \mathrm{C}(\mathrm{X})$, the set of all continuous functions on $\mathrm{X}$. Since all continuous functions are $S$-LSC, therefore $\mathrm{t} 1_{\mathrm{X}} \in \mathcal{H}$. 
Let $\left\{\mathrm{f}_{\alpha}\right\}$ be an arbitrary family of $S$-LSC functions. Then by Theorem 2.11, we have that $\sup \left(\mathrm{f}_{\alpha}\right)$ is also an $S$-LSC function. Hence, $\sup _{\alpha}\left(\mathrm{f}_{\alpha}\right) \in \mathcal{H}$.

Let $\mathrm{f}_{1}, \mathrm{f}_{2} \ldots \mathrm{f}_{\mathrm{n}} \in \mathscr{H}$. Then by Theorem $2.12, \inf _{\mathrm{i}}\left(\mathrm{f}_{\mathrm{i}}\right), \mathrm{i}=1,2 \ldots \mathrm{n}$ also belongs to $\mathscr{H}$.

Thus, the family $\mathscr{H}$ of all $S$-LSC functions from $(\mathrm{X}, \mathrm{T})$ to I satisfies all the conditions to be a fuzzy topology. Thus the family $\mathscr{H}$ forms a fuzzy topology on $\mathrm{X}$.

The Theorem 3.1 is very important, since it implies that the collection of CLSC, $\delta$ LSC, SLSC, etc. functions generate induced stratified fuzzy topologies. The aim of this section is to study the spaces induced by SLSC functions.

Definition 3.2. The fuzzy topology formed by the collection of all SLSC functions from a topological space $(\mathrm{X}, \mathrm{T})$ to the closed unit interval $\mathrm{I}$ is called strongly induced fuzzy topological space or s-induced fts in short and is denoted by $(\mathrm{X}, \mathrm{S}(\mathrm{T}))$.

Now, we cite some examples of this newly defined s-induced fts.

Example 3.3. Let us consider the uncountable non-discrete P-space $\mathrm{S}$ given in Gillman and Jerrison (4N, [20]). In S, all points are isolated except for a distinguished point $\mathrm{s}$, a nbd. of $\mathrm{s}$ being any set containing $\mathrm{s}$ whose complement is countable. Again, let us consider an nbd. $G_{a}, a \in \Lambda$ of $s$. Then $G_{a}$ being a clopen (open and closed) set is regular $\mathrm{F}_{\sigma}$-subset. Let us define $\mathrm{f}_{\mathrm{a}}: \mathrm{S} \rightarrow \mathrm{R}$ by

$$
\begin{aligned}
f_{a}(x) & =1 \text { if } x \in G_{a} \\
& =0 \text { if } x \in S-G_{a} .
\end{aligned}
$$

Each $f_{a}$ is the characteristic function of a regular $F_{\sigma}$-subset is SLSC.

Now, we construct a family $\mathscr{H}$ consisting of all $\mathrm{f}_{\mathrm{a}}$ 's and further let

$$
\mathscr{H}^{*}=\left\{\mathrm{rf}_{\mathrm{a}}: \mathrm{f}_{\mathrm{a}} \in \mathscr{H}, 0 \leq \mathrm{r} \leq 1, \mathrm{a} \in \Lambda\right\} .
$$

Therefore, $\mathscr{H}^{*}$ is a collection of SLSC functions on S, such that

$$
0\left(\equiv \mu_{\phi}\right), 1\left(\equiv \mu_{\mathrm{S}}\right) \in \mathscr{H}^{*}
$$


Considering an arbitrary family $\left\{r_{\alpha} f_{a \alpha}\right\}$ of members of $\mathscr{H}^{*}$, we get that $\vee_{\alpha} r_{\alpha} f_{a \alpha}=$ $\mathrm{f}$ (say) is defined by

$$
\begin{aligned}
f(x) & =\vee_{\alpha} r_{\alpha} \text { if } x \in \bigcup_{\alpha} G_{a \alpha} \\
& =0 \quad \text { if } x \in S-\bigcup_{\alpha} G_{a \alpha} .
\end{aligned}
$$

Clearly, $\cup G_{a}$ is open and closed (all the points of $S-\bigcup_{\alpha} G_{a \alpha}$ are isolated points and $\cup G_{a \alpha}$ is an nbd. of s) and hence $\cup G_{a}$ is a regular $F_{\sigma}$-subset. Thus, $f \in \mathscr{H}^{*}$.

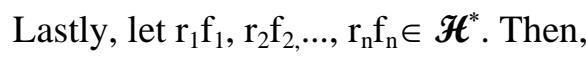

$$
\begin{aligned}
\wedge_{i} r_{i} f_{i} & =\wedge_{i} r_{i} \text { if } x \in \bigcap_{i} G_{a i} \\
& =0 \quad \text { if } x \in S-\bigcap_{i} G_{a i}
\end{aligned}
$$

Clearly, $\wedge_{\mathrm{i}} \mathrm{r}_{\mathrm{i}} \mathrm{f}_{\mathrm{i}}$ belongs to $\mathscr{H}^{*}$.

Thus we see that $\left(\mathrm{S}, \mathfrak{H}^{*}\right)$ forms an s-induced fts.

Example 3.4. Let $(X, T)$ be an indiscrete topological space, i.e. $T=\{\phi, X\}$ and let $\mathrm{f}:(\mathrm{X}, \mathrm{T}) \rightarrow \mathrm{I}$ be a continuous function. Since continuous functions from an indiscrete space to I must be constant, $\mathrm{f}$ is constant. In fact $\mathrm{f}$ is SLSC. Let $\mathrm{x}_{0} \in \mathrm{X}$ and $\varepsilon>0$. Then for all $\mathrm{x} \in \mathrm{X}, \mathrm{f}(\mathrm{x})>\mathrm{f}\left(\mathrm{x}_{0}\right)-\varepsilon$. Since $X$ is a regular $\mathrm{F}_{\sigma}$-subset, $\mathrm{f}$ is SLSC. Let $\mathcal{F}=\{\mathrm{f}$ : $\mathrm{f}$ is SLSC on $\mathrm{X}\}$. Then $(\mathrm{X}, \mathcal{F})$ is a fuzzy topological space and hence an s-induced fts.

Example 3.5. Let $X$ be the closed unit interval $I=[0,1]$. For each natural number $n$, let us define $\mu_{\mathrm{n}} \mathrm{X} \rightarrow \mathrm{I}$ as follows:

$$
\begin{aligned}
\mu_{\mathrm{n}}(\mathrm{x}) & =1 \text { for } \mathrm{x} \in\left[0, \frac{1}{n}\right] \\
& =0 \text { for } \mathrm{x} \in\left(\frac{1}{n}, 1\right] .
\end{aligned}
$$

Let $\mathrm{x}_{0} \in \mathrm{I}$ and $\varepsilon>0$ be arbitrary. Then for all $\mathrm{x} \in\left(0, \frac{1}{n}\right), \mu_{\mathrm{n}}(\mathrm{x})>\mu_{\mathrm{n}}\left(\mathrm{x}_{0}\right)-\varepsilon$. Since $\left(0, \frac{1}{n}\right)$ is regular $\mathrm{F}_{\sigma}$, therefore $\mu_{\mathrm{n}}$ is SLSC. Now we construct $\mathscr{H}=\left\{\mathrm{r} \mu_{\mathrm{n}}: 0 \leq \mathrm{r} \leq\right.$ 1). Similar to Example 3.3, we can show that $\mathscr{H}$ generates a fuzzy topology on I and hence $\{\mathrm{I}, \mathscr{H}\}$ is an s-induced fts. 
Definition 3.6. Let $\alpha$ be a fuzzy subset of a set $X$. Then the crisp subset $\sigma_{\mathrm{r}}(\alpha)$ is called strong r-cut of $\alpha$ iff $\sigma_{\mathrm{r}}(\alpha)=\{\mathrm{x} \in \mathrm{X}: \alpha(\mathrm{x})>\mathrm{r}\}$ for $\mathrm{r} \in \mathrm{I}$.

Theorem 3.7. A fuzzy subset $\lambda$ in an s-induced fts $(X, S(T))$ is open iff for each $r \in I$, the strong $r$-cut $\sigma_{r}(\lambda)$ is a union of regular $F_{\sigma}$-subsets in the topological space $(X, T)$.

Proof. Let the fuzzy subset $\lambda$ be open in (X, S(T)). Then $\lambda \in \mathrm{S}(\mathrm{T})$ i.e. $\lambda$ is an SLSC function. Therefore, for each $r \in R$, the set $\{x \in X: \lambda(x)>r\}$ is a union of regular $F_{\sigma}$-subsets, i.e. $\sigma_{r}(\lambda)$ is a union of regular $F_{\sigma}$-subsets.

Conversely, let the strong r-cut of a fuzzy set $\lambda \sigma_{\mathrm{r}}(\lambda)$ be a union of regular $F_{\sigma^{-}}$ subsets of $X$. Then for each $r \in R,\{x \in X: \lambda(x)>r\}$ is a union of regular $F_{\sigma^{-}}$ subsets. So by Theorem 2.6, $\lambda$ is SLSC and hence $\lambda \in \mathrm{S}(\mathrm{T})$. Thus $\lambda$ is open in $(\mathrm{X}, \mathrm{S}(\mathrm{T}))$.

In a similar way, using Theorem 2.7, it can be proved that the Theorem 3.2 in [5] is not true and the modified form of the theorem is given by:

Theorem 3.8. A fuzzy subset $\lambda$ in a completely induced fts $(X, C(T))$ is open iff for each $r \in I$, the strong $r$-cut $\sigma_{r}(\lambda)$ is a union of regular open subsets in the topological space $(\mathrm{X}, \mathrm{T})$.

Corollary 3.9. If $\sigma_{\mathrm{r}}(\lambda)$ is a regular $F_{\sigma}$-subset, then $\lambda$ is SLSC but the converse is not true, which may be shown by the following example:

Example 3.10. Let us again consider the uncountable non-discrete P-space as taken in Example 3.3. As all the points of $\mathrm{S}$, except a distinguished point $\mathrm{s}$, are isolated, for every point $\mathrm{q} \in \mathrm{S}-\{\mathrm{s}\}$ the set $\{\mathrm{q}\}$ is a regular $\mathrm{F}_{\sigma}$-subset of $\mathrm{S}$. But their union $S-\{s\}$ is not a regular $F_{\sigma^{-}}$-subset of $S$, as $\{s\}$ is not a regular $G_{\delta^{-}}$ subset of $S$ (in fact $\{s\}$ is not even $G_{\delta}$, since in a P-space every $G_{\delta}$ is open (4J, [20]). Now, let us define a function $\mathrm{f}: \mathrm{S} \rightarrow \mathrm{R}$ as follows:

$$
\begin{aligned}
f(x) & =0, \text { if } x=s \\
& =1, \text { otherwise. }
\end{aligned}
$$

We shall now show that $\mathrm{f}$ is SLSC. Let us consider a point $\mathrm{q} \in \mathrm{S}-\{\mathrm{s}\}$. Then $\mathrm{f}(\mathrm{q})$ $=1$ and $\{q\}$ is a regular $F_{\sigma}$-nbd. of $q$ such that for each $\varepsilon>0, f(x)>f(q)-\varepsilon$ for all $x \in\{q\}$. Again, any subset of $S$ containing $s$, say $G$, whose complement is countable, is open and closed and hence a regular $F_{\sigma}$-nbd. of s. Clearly for all $\mathrm{x} \in \mathrm{G}$ and $\varepsilon>0, \mathrm{f}(\mathrm{x})>\mathrm{f}(\mathrm{s})-\varepsilon$. 
Thus $\mathrm{f}$ is SLSC. But for $\mathrm{r}>0, \sigma_{\mathrm{r}}(\mathrm{f})=\{\mathrm{x} \in \mathrm{S}: \mathrm{f}(\mathrm{x})>\mathrm{r}\}=\mathrm{S}-\{\mathrm{s}\}$, which is not a regular $F_{\sigma}$-subset of $S$ but is a union of regular $F_{\sigma}$-subsets.

Note 3.11. The above example also shows that "the necessary and sufficient condition for a real valued function $f$ to be CLSC is that for all $a \in R$, the set $\{\mathrm{x} \in \mathrm{X}: \mathrm{f}(\mathrm{x})>\mathrm{a}\}$ is regular open" is not true, since the function $\mathrm{f}$ defined in the above example is also CLSC and $\{\mathrm{x} \in \mathrm{X}: \mathrm{f}(\mathrm{x})>\mathrm{a}, \mathrm{a}>0\}=\mathrm{S}-\{\mathrm{s}\}$ is not regular open but is a union of regular open subsets (each point of $\mathrm{S}$ other than $\mathrm{s}$ is open and closed).

It can be inferred from Mack [21] that the inverse image of a regular $\mathrm{F}_{\sigma}$-subset under a continuous mapping is again a regular $F_{\sigma}$-subset. Now we define two weaker forms of continuous mapping which will be used in the sequel.

Definition 3.12. A mapping f: $\mathrm{X} \rightarrow \mathrm{Y}$ is said to be s-continuous if the inverse image of every regular $F_{\sigma}$-subset of $Y$ is open in $X$.

Definition 3.13 [13]. A mapping f: $\mathrm{X} \rightarrow \mathrm{Y}$ is said to be $\sigma$-continuous if the inverse image under $f$ of any regular $F_{\sigma}$-subset of $Y$ is a regular $F_{\sigma}$-subset of $X$.

Since, every regular $F_{\sigma}$-subset is an open set, clearly every $\sigma$-continuous mapping is s-continuous.

There is a natural way to associate a fuzzy topology with a given topology and vice versa. Let us denote the collection of all topologies and fuzzy topologies on a non-empty set $\mathrm{X}$ by $\mathcal{T}(\mathrm{X})$ and $\mathscr{F}(\mathrm{X})$ respectively. On the real line $\mathrm{R}$, we take the lower limit topology consisting of the sets of the form $\{(a, \infty): a \in R\}$ together with the empty set $\phi$. The closed unit interval $\mathrm{I}=[0,1]$ will be equipped with the subspace topology denoted by $\mathrm{I}_{\mathrm{r}}$.

Lowen [3] defined two important functors $\mathrm{t}$ and $\omega$ as follows.

The two mappings $\omega: \mathcal{T}(\mathrm{X}) \rightarrow \mathscr{F}(\mathrm{X}): \mathrm{T} \rightarrow \omega(\mathrm{T})$ and $\mathrm{t}: \mathcal{F}(\mathrm{X}) \rightarrow \mathcal{T}(\mathrm{X}): \Lambda \rightarrow \mathrm{t}(\Lambda)$, where $\omega(\mathrm{T})$ and $t(\Lambda)$ are respectively the set of all continuous functions from $(\mathrm{X}, \mathrm{T})$ to $\mathrm{I}_{\mathrm{r}}$ and the initial topology on $\mathrm{X}$ for the family of functions $\Lambda$ and the topological space $I_{r}$.

It is trivial to check that the members of $\omega(\mathrm{T})$ are actually the lower semicontinuous functions when considered as from $(X, T)$ to $I$, where $I=[0,1]$ is equipped with usual topology. 
In the present setting, $\omega(\mathrm{T})$ will be the set of all strongly continuous functions from $(X, T)$ to $I_{r}$ and thus the members of $\omega(T)$ will be the SLSC functions when considered as from $(X, T)$ to $I$, where $I=[0,1]$ is equipped with usual topology.

It can be easily seen that the relations between the two functors $\imath$ and $\omega$ for LSC functions given in Proposition 2.1of [3] are also valid in our present setting for SLSC functions.

In the next two theorems, we discuss the relationships between two topological spaces and their corresponding induced spaces via the s-continuity and $\sigma$ continuity defined above.

Theorem 3.14. If a mapping f: $\left(\mathrm{X}, \mathrm{S}\left(\mathrm{T}_{1}\right)\right) \rightarrow\left(\mathrm{Y}, \mathrm{S}\left(\mathrm{T}_{2}\right)\right)$ is fuzzy continuous, then the mapping $\mathrm{f}:\left(\mathrm{X}, \mathrm{T}_{1}\right) \rightarrow\left(\mathrm{Y}, \mathrm{T}_{2}\right)$ is s-continuous.

Proof. Let $\mathrm{f}:\left(\mathrm{X}, \mathrm{S}\left(\mathrm{T}_{1}\right)\right) \rightarrow\left(\mathrm{Y}, \mathrm{S}\left(\mathrm{T}_{2}\right)\right)$ be fuzzy continuous mapping and let $\mathrm{B}$ be a regular $\mathrm{F}_{\sigma}$-subset of $\left(\mathrm{Y}, \mathrm{T}_{2}\right)$.

Now, $f^{-1}(B)=\{x \in X: f(x) \in B\}$

$=\left\{\mathrm{x} \in \mathrm{X}: \mu_{\mathrm{B}} \mathrm{f}(\mathrm{x})=1\right\}$ (where $\mu_{\mathrm{B}}$ is the characteristic function of the crisp set $\mathrm{B})$

$$
\begin{aligned}
& =\left\{\mathrm{x} \in \mathrm{X}: \mu_{\mathrm{B}} \mathrm{f}(\mathrm{x})>\mathrm{r}, 0 \leq \mathrm{r}<1\right\} \\
& =\left\{\mathrm{x} \in \mathrm{X}:\left(\mathrm{f}^{-1}\left(\mu_{\mathrm{B}}\right)\right) \mathrm{X}>\mathrm{r}, 0 \leq \mathrm{r}<1\right\} \\
& =\sigma_{\mathrm{r}}\left(\mathrm{f}^{-1} \mu_{\mathrm{B}}\right) .
\end{aligned}
$$

$\mu_{\mathrm{B}}$, being the characteristic function of a regular $\mathrm{F}_{\sigma^{-}}$-subset of $\mathrm{Y}$, is an SLSC function, i.e. $\mu_{\mathrm{B}} \in \mathrm{S}\left(\mathrm{T}_{2}\right)$. Therefore, by our assumption, $\mathrm{f}^{-1}\left(\mu_{\mathrm{B}}\right) \in \mathrm{S}\left(\mathrm{T}_{1}\right)$. Hence, by Theorem 3.7, $\mathrm{f}^{-1}(\mathrm{~B})=\sigma_{\mathrm{r}}\left(\mathrm{f}^{-1} \mu_{\mathrm{B}}\right)$ is a union of regular $\mathrm{F}_{\sigma^{-}}$-subsets of $\left(\mathrm{X}, \mathrm{T}_{1}\right)$ and hence open in $\left(\mathrm{X}, \mathrm{T}_{1}\right)$. So $\mathrm{f}$ is s-continuous.

In the reverse direction, we have the following theorem.

Theorem 3.15. A mapping f: $\left(X, S\left(T_{1}\right)\right) \rightarrow\left(Y, S\left(T_{2}\right)\right)$ is fuzzy continuous if the mapping $\mathrm{f}:\left(\mathrm{X}, \mathrm{T}_{1}\right) \rightarrow\left(\mathrm{Y}, \mathrm{T}_{2}\right)$ is $\sigma$-continuous.

Proof. Let $\mathrm{f}:\left(\mathrm{X}, \mathrm{T}_{1}\right) \rightarrow\left(\mathrm{Y}, \mathrm{T}_{2}\right)$ be a $\sigma$-continuous mapping. Also let $\beta$ be an open subset of $\left(\mathrm{Y}, \mathrm{S}\left(\mathrm{T}_{2}\right)\right)$. Now, for $0<\mathrm{r}<1$,

$$
\sigma_{\mathrm{r}}\left(\mathrm{f}^{-1}(\beta)\right)=\left\{\mathrm{x} \in \mathrm{X}:\left(\mathrm{f}^{-1}(\beta)\right) \mathrm{X}>\mathrm{r}\right\}=\{\mathrm{x} \in \mathrm{X}: \beta(\mathrm{f}(\mathrm{x}))>\mathrm{r}\}=(\beta \mathrm{f})^{-1}(\mathrm{r}, 1]
$$




$$
=\mathrm{f}^{-1} \beta^{-1}(\mathrm{r}, 1]=\mathrm{f}^{-1}\left(\beta^{-1}(\mathrm{r}, 1]\right)=\mathrm{f}^{-1}\left(\sigma_{\mathrm{r}}(\beta)\right) .
$$

Now, since $\beta \in \mathrm{S}\left(\mathrm{T}_{2}\right)$, therefore, $\sigma_{\mathrm{r}}(\beta)=\{\mathrm{y} \in \mathrm{Y}: \beta(\mathrm{y})>\mathrm{r}\}$ is a union of regular $\mathrm{F}_{\sigma}$-subsets of $Y$. Again, since $\mathrm{f}$ is $\sigma$-continuous, so $\mathrm{f}^{-1}\left(\sigma_{\mathrm{r}}(\beta)\right)$ is also a union of regular $F_{\sigma}$-subsets of $\left(X, T_{1}\right)$, which in turn implies that $f^{-1}(\beta)$ is SLSC. Hence, $f$ is fuzzy continuous.

Before proceeding further, we recall the concept of initial and final fuzzy topology.

The initial fuzzy topology on $\mathrm{X}$, for the family of fuzzy topological spaces $\left(\mathrm{Y}_{\mathrm{a}}\right.$, $\left.\mathcal{F}_{\mathrm{a}}\right), \mathrm{a} \in \Lambda$ and the family of functions $\mathrm{f}_{\mathrm{a}}: \mathrm{X} \rightarrow\left(\mathrm{Y}_{\mathrm{a}}, \mathcal{F}_{\mathrm{a}}\right)$ is the smallest fuzzy topology on $\mathrm{X}$ making each function $\mathrm{f}_{\mathrm{a}}$ fuzzy continuous and is denoted by $\bigcup_{\mathrm{a} \in \Lambda} \mathrm{f}_{\mathrm{a}}^{-1}\left(\mathcal{F}_{\mathrm{a}}\right)$. Similarly, the final fuzzy topology on $\mathrm{Y}$, for the family of fuzzy topological spaces $\left(\mathrm{X}_{\mathrm{a}}, \mathcal{F}_{\mathrm{a}}\right), \mathrm{a} \in \Lambda$ and the family of functions $\mathrm{f}_{\mathrm{a}}:\left(\mathrm{X}_{\mathrm{a}}, \mathcal{F}_{\mathrm{a}}\right) \rightarrow \mathrm{Y}$ is the finest fuzzy topology on $\mathrm{Y}$ making each function $\mathrm{f}_{\mathrm{a}}$ fuzzy continuous and is denoted by $\cap_{\mathrm{a} \in \Lambda} \mathrm{f}_{\mathrm{a}}\left(\mathcal{F}_{\mathrm{a}}\right)$ [22].

Lemma 3.16 [13]. Let $\mathrm{X}$ be a set, and let $(\mathrm{Y}, \mathrm{T})$ be a topological space and let $\mathrm{f}$ : $\mathrm{X} \rightarrow \mathrm{Y}$ be a map. Then for each SLSC function $\psi$ on $\left(X, \mathrm{f}^{-1}(\mathrm{~T})\right)$ and every $\mathrm{r} \in \mathrm{I}$ (here $\mathrm{f}^{-1}(\mathrm{~T})$ is the initial topology on $\mathrm{X}$ ), it follows that $\psi=\vee_{\mathrm{r}} \mathrm{r} \mu_{\mathrm{A}}$, where $\mathrm{A}=$ $\sigma_{\mathrm{r}}(\psi)$.

Lemma 3.17. If $f: X \rightarrow Y$ is continuous and $B$ is a regular $F_{\sigma}$-subset of $Y$, then $\mathrm{f}^{-1}(B)$ is a regular $F_{\sigma}$-subset of $X$.

Proof. Mack [21] proved that the inverse image of a regular $\mathrm{G}_{\delta}$-subset under a continuous mapping is again regular $\mathrm{G}_{\delta}$. The lemma follows from this result.

Next we consider the relationships between the induced fuzzy topology of the initial topology on $X$ and the initial fuzzy topology of the induced fuzzy topology on Y. For this, we need to define the crisp powerset operators and fuzzy powerset operators.

Definition 3.18. For a given mapping $\mathrm{f}: \mathrm{X} \rightarrow \mathrm{Y}$, the crisp powerset operators $\mathrm{f}^{\rightarrow}: \mathcal{P}(\mathrm{X}) \rightarrow \mathscr{P}(\mathrm{Y})$ and $\mathrm{f} \leftarrow: \mathscr{P}(\mathrm{X}) \leftarrow \mathscr{P}(\mathrm{Y})$ are defined by $\mathrm{f} \rightarrow(\mathrm{A})=\{\mathrm{f}(\mathrm{x}): \mathrm{x} \in \mathrm{A}\}$ and $\mathrm{f}^{\leftarrow}(\mathrm{B})=\{\mathrm{x}: \mathrm{f}(\mathrm{x}) \in \mathrm{B}\}$.

It may be mentioned here that $\mathrm{f} \leftarrow$ is the map adjoint to (in the sense of partially ordered set) $f \rightarrow[23]$. 
Definition 3.19. For a given mapping $\mathrm{f}: \mathrm{X} \rightarrow \mathrm{Y}$, the fuzzy powerset operators $\mathrm{f}_{\mathrm{I}} \rightarrow: \mathrm{I}^{\mathrm{X}} \rightarrow \mathrm{I}^{\mathrm{Y}}$ and $\mathrm{f} \leftarrow: \mathrm{I}^{\mathrm{X}} \leftarrow \mathrm{I}^{\mathrm{Y}}$ are defined by $\mathrm{f}_{\mathrm{I}} \rightarrow(\lambda)(\mathrm{y})=\mathrm{V}\{\lambda(\mathrm{x}): \mathrm{f}(\mathrm{x})=\mathrm{y}\}$ and $\mathrm{f}_{\mathrm{I}} \leftarrow(\delta)(\mathrm{x})=\delta(\mathrm{f}(\mathrm{x})$, where $\lambda$ and $\delta$ are respectively fuzzy subsets of $\mathrm{X}$ and $\mathrm{Y}$.

Theorem 3.20. Let $\mathrm{X}$ be a set and let (Y, $\mathrm{T}$ ) be a topological space. If $\mathrm{f}: \mathrm{X} \rightarrow \mathrm{Y}$ is a mapping, then $\mathrm{f}_{I}^{\leftarrow}(\mathrm{S}(\mathrm{T})) \subseteq \mathrm{S}\left(\mathrm{f}^{\leftarrow}(\mathrm{T})\right)$.

Proof. Let $\theta \in \mathrm{f}_{\mathrm{I}}^{\leftarrow}(\mathrm{S}(\mathrm{T}))$. Then $\theta=\mathrm{f}_{\mathrm{I}}^{\leftarrow}(\delta)$ for some $\delta \in \mathrm{S}(\mathrm{T})$.

Now, $\sigma_{\mathrm{r}}(\delta)=\{\mathrm{y} \in \mathrm{Y}: \delta(\mathrm{y})>\mathrm{r}\}$ is a union of regular $\mathrm{F}_{\sigma^{-}}$-subset of $\mathrm{Y}$ for each $\mathrm{r} \in \mathrm{I}$ (by Theorem 3.6). So, $f \leftarrow\left(\sigma_{\mathrm{r}}(\delta)\right)$ is also a union of regular $F_{\sigma}$-subset of $X$ (by Lemma 3.17).

Again, $\sigma_{\mathrm{r}}(\theta)=\sigma_{\mathrm{r}}\left(\mathrm{f}_{\mathrm{I}}^{\leftarrow}(\delta)\right)=\mathrm{f}^{\leftarrow}\left(\sigma_{\mathrm{r}}(\delta)\right)$ (Lemma 2.2 (i) of [4]), which is a union of regular $F_{\sigma}$-subsets of $\left(X, f^{\leftarrow}(T)\right)$, since $f$ is continuous. Again by Theorem 3.7, $\theta \in \mathrm{S}\left(\mathrm{f}^{\leftarrow}(\mathrm{T})\right)$.

Hence, $\mathrm{f}_{\mathrm{I}}^{\leftarrow}(\mathrm{S}(\mathrm{T})) \subseteq \mathrm{S}\left(\mathrm{f}^{\leftarrow}(\mathrm{T})\right)$.

For the converse we have the following theorem.

Theorem 3.21. Let $\mathrm{X}$ be a set and let $(\mathrm{Y}, \mathrm{T})$ be a topological space such that every member of $\mathrm{T}$ is a union of regular $\mathrm{F}_{\sigma}$-subsets of $(\mathrm{Y}, \mathrm{T})$. If $\mathrm{f}: \mathrm{X} \rightarrow \mathrm{Y}$ is a mapping, then $S\left(f^{\leftarrow}(T)\right) \subseteq f_{I}^{\leftarrow}(S(T))$.

Proof. Let $\psi \in \mathrm{S}\left(\mathrm{f}{ }^{\leftarrow}(\mathrm{T})\right)$, so that for each $\mathrm{r} \in \mathrm{I}, \sigma_{\mathrm{r}}(\psi)=\{\mathrm{x} \in \mathrm{X}: \psi(\mathrm{x})>\mathrm{r}\}$ is a union of regular $\mathrm{F}_{\sigma}$-subsets of $\left(X, f^{\leftarrow}(T)\right)$ and thus is open. Therefore, $\sigma_{r}(\psi) \in f$

$\leftarrow(T)$. Hence, there exists $B_{r} \in T$ such that $\sigma_{r}(\psi)=f \leftarrow\left(B_{r}\right)$. Again since $B_{r} \in T$, so $B_{r}$ is a union of regular $F_{\sigma}$-subsets of $(Y, T)$.

Let us consider the function $\beta: \mathrm{Y} \rightarrow \mathrm{I}$ defined by $\beta=\vee_{\mathrm{r} \in \mathrm{I}} \mathrm{r} \mu_{\mathrm{Br}}$.

We consider the set $\sigma_{\mathrm{s}}(\beta)=\{\mathrm{y} \in \mathrm{Y}: \beta(\mathrm{y})>\mathrm{s}\}, \mathrm{s} \in \mathrm{I}$. It follows that

$$
\sigma_{s}(\beta)=\bigcup_{r}>{ }_{s} B_{r} \text {, which is clearly a union of regular } F_{\sigma} \text {-subsets of } Y \text {. }
$$

Now, $\mathrm{f}^{\leftarrow}(\beta)=\mathrm{f}^{\leftarrow}\left(\vee_{\mathrm{r}} \mathrm{r} \mu_{\mathrm{Br}}\right)=\vee_{\mathrm{r}}\left(\mathrm{f}^{\leftarrow}\left(\mathrm{r} \mu_{\mathrm{Br}}\right)\right)$ (by Lemma 2.2(iv) of [4])

$$
\begin{aligned}
& \left.=\vee_{\mathrm{r}} \mathrm{r} \mu_{\mathrm{f}} \leftarrow{ }_{(\mathrm{Br}}\right)(\text { by Lemma 2.2(iii) of [4]) } \\
& =\vee_{\mathrm{r}} \mathrm{r} \mu_{\mathrm{A}}, \mathrm{A}=\sigma_{\mathrm{r}}(\psi)
\end{aligned}
$$


$=\psi($ by Lemma 3.16).

Thus, $\psi \in \mathrm{f}_{\mathrm{I}}^{\leftarrow}(\mathrm{S}(\mathrm{T}))$, i.e., $\psi \in \mathrm{S}\left(\mathrm{f}_{\mathrm{I}}^{\leftarrow}(\mathrm{T})\right)$ implies $\psi \in \mathrm{f}_{\mathrm{I}}^{\leftarrow}(\mathrm{S}(\mathrm{T}))$.

Hence, $\mathrm{S}\left(\mathrm{f}^{\leftarrow}(\mathrm{T})\right) \subseteq \mathrm{f}_{\mathrm{I}} \leftarrow(\mathrm{S}(\mathrm{T}))$

Theorem 3.22. If $f_{n}:\left(X_{n}, T_{n}\right) \rightarrow Y, n \in N$ are functions from a family of topological spaces $\left\{\left(\mathrm{X}_{\mathrm{n}}, \mathrm{T}_{\mathrm{n}}\right)\right\}$ onto a set $\mathrm{Y}$, then $\mathrm{S}\left(\cap_{\mathrm{n} \in \mathrm{N}} \mathrm{f}_{\mathrm{n}} \rightarrow\left(\mathrm{T}_{\mathrm{n}}\right)\right) \subset$ $\cap_{n \in N}\left(f_{n}\right)_{I} \rightarrow\left(S\left(T_{n}\right)\right)$, where $\left(\cap_{n \in N} f_{n} \rightarrow\left(T_{n}\right)\right)$ is the final topology on $Y$.

Proof. Let $\lambda \in S\left(\cap_{n \in N} f_{n} \rightarrow\left(T_{n}\right)\right)$.

Then, for each $r \in I, \sigma_{r}(\lambda)$ is a union of regular $F_{\sigma}$-subsets in $\bigcap_{n \in N} f_{n} \rightarrow\left(T_{n}\right)$.

This implies that $\sigma_{r}(\lambda)$ is a union of regular $F_{\sigma}$-subsets in each $f_{n} \rightarrow\left(T_{n}\right)$. Thus $f_{n} \leftarrow\left(\sigma_{r}(\lambda)\right)$ is also a union of regular $F_{\sigma}$-subsets in $\left(X_{n}, T_{n}\right)$ for each $n$.

Now, $f_{n} \leftarrow\left(\sigma_{r}(\lambda)\right)=\sigma_{r}\left(f_{n} \leftarrow(\lambda)\right)$ (Lemma 2.2 (i) of [24]). Therefore, $\sigma_{r}\left(f_{n} \leftarrow(\lambda)\right)$ is a union of regular $F_{\sigma}$-subsets of $\left(X_{n}, T_{n}\right)$ for each $n \in N$. So, by Theorem 3.7, $\left(f_{n}\right)_{I}^{\leftarrow}(\lambda) \in S\left(T_{n}\right)$ for each $n$.

Hence $\lambda \in\left(f_{n}\right)_{I} \rightarrow\left(S\left(T_{n}\right)\right)$ for each $n \in N$, since $f_{n}$ is onto.

As a result, $\lambda \in \bigcap_{n \in N}\left(f_{n}\right)_{I} \rightarrow\left(S\left(T_{n}\right)\right)$. This completes the proof.

As a particular case, when $n=1$, we have the following result.

Corollary 3.23. Let $(X, S(T))$ be the s-induced fts on the topological space $(\mathrm{X}, \mathrm{T})$. For a set $\mathrm{Y}$, if $\mathrm{f}:(\mathrm{X}, \mathrm{T}) \rightarrow \mathrm{Y}$ is an onto mapping, then $\mathrm{S}(\mathrm{f} \rightarrow(\mathrm{T})) \subset$ $\mathrm{f}_{\mathrm{I}} \rightarrow(\mathrm{S}(\mathrm{T}))$, where $\mathrm{f} \rightarrow(\mathrm{T})$ is the final topology on $\mathrm{Y}$.

So far, we have discussed some of the important properties of the induced fuzzy topological space $(\mathrm{X}, \mathrm{S}(\mathrm{T}))$ and the nature of relationship between the induced fuzzy topological space (X, S(T)) generated by SLSC functions and the underlying topological space $(\mathrm{X}, \mathrm{T})$. Now, it is natural to ask, when will a fuzzy topological space be an s-induced fuzzy topological space? To conclude, we put forward the following theorems in this direction.

Definition 3.24. Let $\alpha$ be a fuzzy subset of a set $X$. Then the crisp subset $\omega_{\mathrm{t}}(\alpha)$ is called a $\mathrm{t}$-cut of $\alpha$ iff $\omega_{\mathrm{t}}(\alpha)=\{\mathrm{x} \in \mathrm{X}: \alpha(\mathrm{x}) \geq \mathrm{t}\}$ for $\mathrm{t} \in \mathrm{I}$. 
Theorem 3.25. Let $(X, \mathcal{F})$ be an s-induced fuzzy topological space and $\mathrm{T}=\mathfrak{F} \cap$ $2^{\mathrm{X}}$ be the crisp members of $\mathcal{F}$. Then the following two statements are equivalent:

For any fuzzy subset $\lambda$ and $r \in I$,

$\omega_{\mathrm{r}}\left(\mathrm{cl}_{\mathcal{F}}(\lambda)\right)=\bigcap\left\{\mathrm{cl}_{\mathrm{T}}\left(\omega_{\mathrm{t}}(\lambda)\right): \mathrm{t}<\mathrm{r}\right\}$ is a regular $\mathrm{G}_{\delta}$-subset in $\mathrm{X}$.

For any fuzzy subset $\mu$ and $\mathrm{p} \in \mathrm{I}$

$\sigma_{\mathrm{p}}\left(\operatorname{int}_{\mathcal{F}}(\mu)\right)=\bigcup\left\{\operatorname{int}_{\mathrm{T}}\left(\sigma_{\mathrm{q}}(\mu)\right): \mathrm{q}>\mathrm{p}\right\}$ is a regular $\mathrm{F}_{\sigma^{-}}$-subset in $\mathrm{X}$.

Proof. The theorem can be proved in a similar fashion as Theorem 2.3 of [25].

Theorem 3.26. Let $(\mathrm{X}, \mathcal{F})$ be a fuzzy topological space and let $\mathrm{T}=\mathscr{F} \cap 2^{\mathrm{X}}$ be the crisp part of $\mathcal{F}$. Then the s-induced fuzzy topology $\mathrm{S}(\mathrm{T})$ on $\mathrm{X}$ is same as the fuzzy topology $\mathcal{F}$ if for any fuzzy subset $\lambda$ and $r, t \in I$

$$
\omega_{\mathrm{r}}\left(\mathrm{cl}_{\mathcal{F}}(\lambda)\right)=\bigcap\left\{\mathrm{cl}_{\mathrm{T}}\left(\omega_{\mathrm{t}}(\lambda)\right): \mathrm{t}<\mathrm{r}\right\} \text { is a regular } \mathrm{G}_{\delta} \text {-subset of }(\mathrm{X}, \mathrm{T}) .
$$

Proof. Let $\lambda$ be a closed subset of $(X, \mathscr{F})$ and $r \in I$. Then, by the given condition

$$
\omega_{\mathrm{r}}(\lambda)=\omega_{\mathrm{r}}\left(\mathrm{cl}_{\mathcal{F}}(\lambda)\right)=\bigcap\left\{\mathrm{cl}_{\mathrm{T}}\left(\omega_{\mathrm{t}}(\lambda)\right): \mathrm{t}<\mathrm{r}\right\} \text { is a regular } \mathrm{G}_{\delta} \text {-subset of }(\mathrm{X}, \mathrm{T}) .
$$

Taking complement, we get $X-\omega_{r}(\lambda)$ is a regular $F_{\sigma}$-subset of $(X, T)$, i.e. $\sigma_{1-r}\left(1_{X}-\lambda\right)$ is a regular $F_{\sigma}$-subset of $(X, T)$, i.e. $\left(1_{X}-\lambda\right)$ is open in $(X, S(T))$, i.e. $\lambda$ is closed in $(X, S(T))$.

Again, let $\mu$ be a closed fuzzy subset in $(X, S(T))$ and $r \in I$. Then, by given condition

$$
\omega_{\mathrm{r}}\left(\operatorname{cl}_{\mathcal{F}}(\mu)\right)=\bigcap\left\{\mathrm{cl}_{\mathrm{T}}\left(\omega_{\mathrm{t}}(\mu)\right): \mathrm{t}<\mathrm{r}\right\} \text { is a regular } \mathrm{G}_{\delta} \text {-subset of }(\mathrm{X}, \mathrm{T}) .
$$

Since $\mu$ is closed in $S(T)$, from the given condition, it can be seen that $\omega_{t}(\mu)$ is a regular $\mathrm{G}_{\delta}$-subset in $(\mathrm{X}, \mathrm{T})$ and hence closed in $(\mathrm{X}, \mathrm{T})$.

Thus, $\mathrm{cl}_{\mathrm{T}}\left(\omega_{\mathrm{t}}(\mu)\right)=\omega_{\mathrm{t}}(\mu)$, and therefore $\omega_{\mathrm{r}}\left(\operatorname{cl}_{\mathcal{F}}(\mu)\right)=\bigcap\left\{\left(\omega_{\mathrm{t}}(\mu)\right): \mathrm{t}<\mathrm{r}\right\}=\omega_{\mathrm{r}}(\mu)$. i.e. $\mu=\operatorname{cl}_{\mathcal{F}}(\mu)$, which shows that $\mu$ is closed in $\mathcal{F}$. 
As a consequence, we get $\mathcal{F}=\mathrm{S}(\mathrm{T})$.

\section{Conclusion}

In this paper, a generalized induced fuzzy topological space has been introduced and its properties were investigated. Its importance lies in the fact that some induced spaces already prevailing in the literature come as special cases of this generalized space. Also some flaws of induced fuzzy spaces already introduced by different authors have been detected and corrected accordingly. On the other hand, as a particular case of the newly introduced generalized space, a new induced fuzzy structure has been defined. The properties of the induced fuzzy space generated by the class of SLSC functions have been studied. The interrelationships between the fuzzy topology and newly defined induced fuzzy topology have been investigated and some interesting results have been obtained. The interested researcher may study the space further and find various applications of this space. Also, it can be used as a tool to study various topological properties in fuzzy setting. Apart from that, other stronger/weaker versions of LSC functions may be defined and possible fuzzy topological structures or their generalized forms may be studied. Also, the suggestions pointed out in this paper regarding some already published results create the scope of study of those spaces under the modifications made. Lastly, this paper poses some interesting open questions, which pave the way for further study, e.g., the equality condition in the Theorem $3.20,3.21,3.22$ and 3.23 may be investigated; different types of functors arising from the different choices of $S$ sets and their interrelationship may be worked out, and also the fuzzy concepts of this paper can be translated into L-fuzzy setting.

\section{Acknowledgements}

The authors express their sincere thanks to the referees for their valuable comments and suggestions towards improvement of the paper.

\section{References}

[1] Chang, C.L., Fuzzy Topological Spaces, J. Math. Anal. Appl., 24, pp. 182-190, 1968.

[2] L.A. Zadeh, Fuzzy Sets, Inform. and control, 8, pp. 338-353, 1965.

[3] R. Lowen, Fuzzy Topological Spaces and Fuzzy Compactness, J. Math. Anal. Appl. 56, pp. 621-633, 1976.

[4] M.D. Weiss, Fixed Points, Separation, and Induced Topologies for Fuzzy Sets, J. Math. Anal. Appl., 50, pp. 142-150, 1975.

[5] Bhaumik, R.N. \& Mukherjee, A., Completely Lower Semi-Continuous Functions, Math. Edu. 26, pp. 66-69, 1992. 
[6] Mukherjee, A. \& Halder, S., $\delta$-Induced Fuzzy Topological Spaces, Proc. Nat. Sem. on Recent Trends in Maths. \& Its Appl., April 28-29, 2003, pp. 177-182, 2003.

[7] Bhaumik, R.N. \& Bhattacharya, D., On Regular Semi-Continuous Functions, Math. Edu., xxvi(1), pp. 11-17, 1992.

[8] Bhaumik, R.N. \& Mukherjee, A., Some More Results on Completely Induced Fuzzy Topological Spaces, Fuzzy Sets and Systems, 50, pp. 113117, 1992.

[9] Monsef, M.E. Abd. El. \& Ramadan, A.E., On Fuzzy Supra Topological Spaces, Indian J. Pure and Appl. Math., 18, pp. 322-329, 1987.

[10] Mukherjee, A., Some More Results on Induced Fuzzy Topological Spaces, Fuzzy Sets and Systems, 96, pp. 255-258, 1998.

[11] Bhaumik, R.N. \& Bhattacharya, D., Regular $G_{\delta}$-Subsets and Real Compact Spaces, Bull. Cal. Math. Soc., 87, pp. 39-44, 1995.

[12] Dilworth, R.P., The Normal Completion of Lattice of Continuous Functions, Trans. Amer. Math. Soc., 68, pp. 427-438, 1950.

[13] Bhattacharjee, D. \& Saha, A.K., Fuzzy Topological Spaces Induced by Regular Lower Semi-Continuous Functions, Proc. Nat. Sem. on Fuzzy Math. \& Its Appl., Nov. 25-26, pp. 47-56, 2006.

[14] Saha, A.K. \& Bhattacharya, D., A Study on Induced Fuzzy Topological Space Generated By M-RLSC Functions, Proceedings of International Conference on Rough Sets, Fuzzy Sets and Soft Computing, Nov. 5-7, pp. 400-408, 2009.

[15] Alimohammady, M. \& Roohi, M., Fuzzy Minimal Structure and Fuzzy Minimal Vector Spaces, Chaos, Solitons and Fractals, 27, pp. 599-605, 2006.

[16] El Naschie, M.S., On The Uncertainty of Cantorian Geometry and The Two-Slit Experiment, Chaos, Solitons and Fractals, 9(3), pp. 517-529, 1998.

[17] El Naschie, M.S., On The Unification of Heterotic Strings, M Theory and $\varepsilon^{(\infty)}$ Theory, Chaos, Solitons and Fractals, 11(14), pp. 2397-2408, 2000.

[18] Kelley, J.L., General Topology, D. Van Nostrand, Princeton, NJ, 1955.

[19] Bhaumik, R.N. \& Mukherjee, A., Completely Induced Fuzzy Topological Spaces, Fuzzy Sets and Systems, 47, pp. 387-390, 1992.

[20] Gillman, L. \& Jerrison, M., Rings of Continuous Function, Van Nostrand, 1960.

[21] Mack, J.E., Countable Paracompactness and Weak Normality Properties, Trans. Amer. Math. Soc., 148, pp. 256-272, 1970.

[22] Palaniappan, N., Fuzzy Topology, Alpha Science International Ltd; 2002.

[23] Rodabaugh, S.E., Powerset Operator Based Foundation for Point-Set Lattice-Theoretic (Poslat) Fuzzy Set Theories and Topologies, Quaest. Math., 20(3), pp. 463-530, 1997. 
[24] Geping, W. \& Lanfang, H., On Induced Fuzzy Topological Space, J. Math. Anal. Appl., 108, pp. 495-506, 1985.

[25] Bhattacharya, D. \& Saha, A.K., A Note On R-Countably Induced Fuzzy Topological Space, Proc. Nat. Sem. On Rec. Dev. in Math. \& Its Appl. Nov. 14-15, pp. 1-5, 2008. 